\title{
An Unusual Case of Community-acquired Aeromonas hydrophila Gastroenteritis Causing Delayed-onset Obstructive Hydrocephalus in a Child after Posterior Fossa Craniotomy for a Tumor: A Case Report and Review of Literature
}

\author{
Santosh M Rao ${ }^{1}$, Nivedhana Subburaju ${ }^{2}$, Nataraj Palaniappan ${ }^{3}$, Vivekanand Vishnampettai Varadarajan ${ }^{4}$
}

\begin{abstract}
A hitherto unusual case of community-acquired Aeromonas hydrophila gastroenteritis causing obstructive hydrocephalus in a patient recovering well after a craniotomy is presented. Earlier cases of $A$. hydrophila causing septicemia and meningitis in neonates, postoperative wound infections after neurotrauma, and after spinal surgery have been reported in the literature.

Keywords: Aeromonas hydrophila, Craniotomy, Meningitis, Ventriculoperitoneal shunt.

Pediatric Infectious Disease (2022): 10.5005/jp-journals-10081-1309
\end{abstract}

\section{Case Description}

A 4-year-old girl presented to us with features of headache, vomiting, and gait unsteadiness of about 4 weeks of duration. The symptoms were insidious in onset and gradually progressive. Clinical examination revealed a GCS of 15/15, PERL, bilateral papilledema, no oculomotor palsies, or weakness. She showed signs of cerebellar dysfunction, namely, gait ataxia and bilateral dysmetria. MRI brain with contrast revealed a cerebellar cystic lesion with obstructive triventricular hydrocephalus. She underwent an emergency burr hole and external ventricular drain (EVD) placement followed by suboccipital midline craniotomy and total excision of the lesion. Post-surgery she was stable. Post-op CT scan brain with contrast done on the 1st postoperative day revealed complete excision of the lesion with resolving hydrocephalus. She tolerated the occlusion of the EVD which was removed after 96 hours. Before removal, a repeat CT brain-plain done on the 4 th postoperative day revealed a further decrease in ventricular size with the restoration of sulci and gyri and resolution of periventricular lucency. The operative site was lax with no pseudomeningocele and she gradually improved. She was discharged on the 10th postoperative day. At the time of discharge, she was fully conscious, afebrile with bilateral cerebellar deficits which were improving. She was sitting in bed and was on oral feeds. The frontal burr hole and the posterior suboccipital craniotomy sites were lax and there was no pseudomeningocele. The CRP was negative and the CSF and blood cultures were sterile. HPE was reported as a pilocytic astrocytoma-Grade 1. She was on regular weekly outpatient follow-up and was doing well. The craniotomy site was lax and the wound was healthy and had healed well.

Four weeks after discharge, she presented to us with drowsiness, irritability, sudden-onset high fever with rigors, diarrhea, and a tense swelling at the back of the neck with an intermittent clear watery discharge of 2 days of duration. The discharge occurred through a small fistula in the lower end of the midline occipital scar which had previously healed completely.
1'Department of Neurosurgery, Rainbow Children's Hospital, Chennai, Tamil Nadu, India

${ }^{2}$ Department of Microbiology, Rainbow Children's Hospital, Chennai, Tamil Nadu, India

${ }^{3}$ Department of Paediatric Intensive Care, Rainbow Children's Hospital, Chennai, Tamil Nadu, India

${ }^{4}$ Department of Paediatrics, Rainbow Children's Hospital, Chennai, Tamil Nadu, India

Corresponding Author: Santosh M Rao, Department of Neurosurgery, Rainbow Children's Hospital, Chennai, Tamil Nadu, India, Phone: +91 9600086948, e-mail: kasamora@gmail.com

How to cite this article: Rao SM, Subburaju N, Palaniappan N, et al. An Unusual Case of Community-acquired Aeromonas hydrophila Gastroenteritis Causing Delayed-onset Obstructive Hydrocephalus in a Child after Posterior Fossa Craniotomy for a Tumor: A Case Report and Review of Literature. Pediatr Inf Dis 2022;4(1):21-23.

Source of support: Nil

Conflict of interest: None

A detailed history revealed that many of the family members including the parents who were primary caregivers had suffered from acute gastroenteritis and diarrheal episodes over the past week. A contaminated drinking water source exposure was postulated to be the probable cause.

\section{INVESTIGATIONS}

\section{CT Brain}

Plain revealed obstructive triventricular hydrocephalus with obliteration of the sulci-gyri pattern.

\section{Lab Investigations}

The initial CRP was $200 \mathrm{mg} / \mathrm{L}$, total count 15,700 cells $/ \mathrm{mm}^{3}$, DC: $\mathrm{N}-77 \%, \mathrm{~L}-17 \%$. Initial blood, urine, and wound discharge (from the pseudomeningocele) cultures were sterile. 


\section{Cerebrospinal Fluid (CSF)}

CSF (obtained from the external ventricular drain): CSF sugar and protein were 54 and $670 \mathrm{mg} / \mathrm{dL}$, respectively. CSF culture showed growth of Aeromonas hydrophila.

Repeat counts (48 hours after antibiotics) were normal, CRP $<10$ was negative, and subsequent CSF cytology was normal, and blood and CSF cultures were sterile.

\section{Microbiological Methodology}

The CSF was straw-colored and showed few pus cells and no organisms in gram stain. Direct culture of CSF was done. The CSF was centrifuged and the sediment was loaded into a BACTEC bottle (automated culture system). Gram-stained smear from the culture bottle flagged positively showed Gram-negative bacilli which were subcultured onto blood, MacConkey, and chocolate agars. The GNB was oxidase-positive and motile. It produced non-hemolytic gray moist colonies on sheep blood agar, gray colonies on chocolate agar, and non-lactose fermenter colonies on MacConkey agar. Biochemical identification tests showed a positive reaction to indole production. Triple sugar iron agar had an alkaline slant, acid butt with no hydrogen sulfide production. Citrate utilization and mannitol fermentation tests were negative. Further testing was done with Vitek-2 since identification could not arrive with biochemical reactions alone. The organism was identified as $A$. hydrophila.

Antibiogram revealed that the isolate was sensitive to cephalosporins, amoxicillin-clavulanic acid, piperacillin-tazobactam, tetracycline, imipenem, meropenem, ciprofloxacin, amikacin, gentamicin, and resistant to chloramphenicol and cotrimoxazole.

\section{TREATMENT}

The child underwent an emergency external ventricular drain placement through a right frontal burr hole. Before starting empirical broad-spectrum antibiotics, blood and CSF cultures were sent. Her diarrhea abated. Her fever subsided. Her wound and blood cultures were negative. The CSF, however, was positive for A. hydrophila. Her CRPs returned to $<10$ and she was afebrile within 48 hours of starting meropenem and vancomycin. She did not tolerate EVD occlusion at all. It was therefore decided that she would need a permanent CSF diversion. The case was discussed by a multidisciplinary team comprising the neurosurgeon, pediatric intensivist, microbiologist, infectious disease specialist, and the admitting pediatrician. It was concluded that as the child had meningitis and also as she was recovering from tumor surgery, the safest and quickest route to restoring normalcy and to her returning home would be the placement of a ventriculoperitoneal shunt once the infection abates.

As a VP shunt meant the placement of indwelling hardware, it was decided in concurrence with the multidisciplinary team to continue meropenem for a total of 14 days. CSF and blood cultures were to be repeated on day 14 .

If the cultures were sterile after 72 hours, a repeat CT brain followed by a VP shunt was planned. The decided treatment course was followed. The repeat cultures sent on day 14 were still sterile and she underwent a VP shunt on day 17. Post shunt she was well and was discharged. She was on regular follow-up. MRI brain with contrast done at 12 weeks showed no tumor recurrence and complete resolution of hydrocephalus. At 6 months of follow-up, the child is active and well. The shunt is working well.

\section{Discussion}

Aeromonas species are oxidase-positive, motile, gram-negative bacilli exhibiting fermentative metabolism. They are ubiquitous in nature, especially in water ecosystems and soil. Aeromonas hydrophila contamination of surface water is an emerging threat to public health by a recent study in Kolkata. ${ }^{1}$ The family Aeromonadaceae includes 14 species in the genus Aeromonas. ${ }^{2,3}$ Four categories of infections have been described: gastroenteritis, cellulitis and wound infections, septicemia, and miscellaneous infections. Liver disease, diabetes, and malignancies make the patient more susceptible to Aeromonas septicemia. Meningitis due to Aeromonas is remarkably rare. Aeromonas sepsis has been reported in pediatric medical literature. Fatal Aeromonas sepsis and meningitis have been reported in a child with sickle cell anemia. ${ }^{4}$ Fulminant Aeromonas sepsis and meningitis in pre-term infants have also been reported..$^{5}$ Aeromonas sepsis can occur in patients with no immunological and physiological deficits but the incidence is $<0.15 \% .{ }^{6}$ Aeromonas meningitis is rare but has no specific age predilection and can be nosocomial ${ }^{7,8}$ or community-acquired. ${ }^{9}$ Multifocal osteomyelitis due to Aeromonas in a child with leukemia has also been reported..$^{10}$ Wound infection due to freshwater contamination by $A$. hydrophila has also been reported. ${ }^{11,12}$

Aeromonas infections in neurosurgery have been reported in a few instances but mostly as a nosocomial infection as in post-traumatic brain abscess ${ }^{13}$ or as brain abscess in pre-term neonates. ${ }^{14}$

Community-acquired $A$. hydrophila meningitis severe enough to cause hydrocephalus has not been reported earlier in the literature to the best of our knowledge. What makes our case unique is the unusual mode of presentation and the fact that the child had recovered from hydrocephalus and was convalescing after a craniotomy for the tumor. The child who had earlier tolerated EVD occlusion and removal after craniotomy could not tolerate EVD occlusion after 4 weeks due to Aeromonas meningitis. We postulate that exposure to a contaminated water source resulted in Aeromonas gastroenteritis. Hematogenous spread then resulted in meningitis and causation of hydrocephalus due to probable aqueductal scarring. The resolving blood and debris post-surgery would have served as a nidus for colonization by Aeromonas. The tense pseudomeningocele would have decompressed through the weakest area of the surgical scar. The rapidity of the sequence of events does make one wonder about the virulence of the organism. The child improved with 14 days of meropenem. Fortunately, the organism was sensitive to a spectrum of antibiotics though multidrug resistance in $A$. hydrophila is not unheard of. ${ }^{15}$

\section{Conclusion}

Aeromonas hydrophila infection though rare is not to be taken lightly. The need for identifying the species is essential to clinch the diagnosis and also to aid in prognostication. The role of automation in microbiology for aiding in the diagnosis of this pathogen needs to be emphasized. Prompt diagnosis and treatment are essential. A clear treatment plan for the infection after discussion with the concerned specialists is the sine qua non. One must not lower one's guard while treating this pathogen. 


\section{References}

1. Bhowmik P, Bag PK, Hajra TK, et al. Pathogenic potential of aeromonas hydrophila isolated from surface waters in Kolkata, India. J Med Microbiology 2009;58(Pt 12):1549-1558. DOI: 10.1099/jmm.0.0143160.

2. Carnahan AM, Behram S, Joseph SW, et al. A flexible key for identifying clinical Aeromonas species. J Clin Microbiol 1991;29(12):2843-2849. DOI: 10.1128/JCM.29.12.2843-2849.1991.

3. Janda JM, Abbott SL. The genus aeromonas: taxonomy, pathogenicity, and infection. Clin Microbiol 2010;Rev. 23(1):35-73. DOI: 10.1128/ CMR.00039-09.

4. Yadava R, Seeler RA, Kalelkar M, et al. Fatal aeromonas hydrophila sepsis and meningitis in a child with sickle cell anemia. Am J Dis Child 1979;133(7):753-754. DOI: 10.1001/archpedi.1979.02130070089023.

5. Kali A, Kalaivani R, Charles PMV, et al. Aeromonas hydrophila meningitis and fulminant sepsis in preterm newborn: a case report and review of literature. Ind J Med Microbiology 2016;34(4):544-547. DOI: 10.4103/0255-0857.195383.

6. Janda JM, Guthertz LS, Kokka RP, et al. Aeromonas species in septicemia: laboratory characteristics and clinical observations. Clin Infect Dis 1994;19(1):77-83. DOI: 10.1093/clinids/19.1.77.

7. Parras F, Diaz MD, Reina J, et al. Meningitis due to aeromonas spp: case report and review. Clin Infect Dis 1993;17(6):1058-1060. DOI: 10.1093/clinids/17.6.1058.
8. Jacob L, Carron DB, Haji TC, et al. An unusual case of pyogenic meningitis due to Aeromonas sobria. Br J Hosp Med 1988;39(5):449.

9. Fratzia JD. Community-acquired Aeromonas hydrophila meningitis. Emerg Med Australas 1993;5(4):251-253. DOI: 10.1111/j.14422026.1993.tb00114.x.

10. Doganis D, Baka M, Tsolia M, et al. Multifocal Aeromonas osteomyelitis in a child with leukemia. Case reports in infectious diseases. 2016;2016:8159048. DOI: 10.1155/2016/8159048.

11. Skiendzielewski JJ, O'Keefe KP. Wound infection due to fresh water contamination by Aeromonas hydrophila. J Emerg Med 1990;8(6):701703. DOI: 10.1016/0736-4679(90)90281-Y.

12. Semel JD, Trenholme G. Aeromonas hydrophila water-associated traumatic wound infections: a review. J Trauma 1990;30(3):324-327. DOI: 10.1097/00005373-199003000-00011.

13. Mahabeer Y, Khumalo A, Kiratu E, et al. Posttraumatic brain abscess caused by Aeromonas hydrophila. J Clin Microbiol 2014;52(5):17961797. DOI: $10.1128 / J C M .00267-14$.

14. Bukhari EE. Unusual multiple brain abscesses caused by Aeromonas hydrophila in a preterm neonate: case report.J Child Sci 2018;8(01):e55e57. DOI: 10.1055/s-0038-1669475.

15. Ugarte-Torres A, Perry S, Franko A, et al. Multidrug-resistant Aeromonas hydrophila causing fatal bilateral necrotizing fasciitis in an immunocompromised patient: a case report. J Med Case Rep 2018;12(1):326. DOI: 10.1186/s13256-018-1854-1. 